\title{
La teoría de agencia: el doble rol y su impacto en la acreditación de los institutos profesionales y centros de formación técnica en Chile
}

\author{
Ezequiel Martínez-Rojas, Ninoscka Zencovich-Burdiles, Alberto Martínez-Quezada y \\ Militza Fernández-Céspedes \\ Universidad Arturo Prat. Av. Arturo Prat 2120, lquique, Chile. (Correo-e: emartinezr@unap.cl; nzencovich@unap.cl; \\ albmarti@unap.cl; milfernand@unap.cl)
}

Recibido Mar. 31, 2021; Aceptado Jun. 5, 2021; Versión final Jun. 23, 2021, Publicado Oct. 2021

\begin{abstract}
Resumen
El presente trabajo tiene por objeto analizar, desde la teoría de agencia, el impacto del fenómeno del doble rol en la acreditación de los institutos profesionales (IP) y centros de formación técnica (CFT) en Chile. Empleando un enfoque de investigación mixto y exploratorio, se inspeccionan los dictámenes de acreditación de 24 IP y 18 CFT, un 62\% del subsistema técnico profesional de Chile. Se constata indicio de doble rol en 7 instituciones (17\%), las que promedian 2.1 años de acreditación, resultado comparativamente menor al conjunto de instituciones acreditadas. Se analizan 12 variables cuantitativas de acreditación institucional, constatando que, la observancia de doble rol aunado a otras cuatro variables, constituyen componentes significantes al momento de explicar los años de acreditación de los IP y CFT chilenos. Se concluye que el fenómeno del doble rol en los IP y CFT es un componente que conduce a tensiones y no garantiza una adecuada separación de funciones (gestión y propiedad), incidiendo negativamente en su acreditación.
\end{abstract}

\section{The agency theory: the dual role and its impact on the accreditation of professional institutes and technical training centers in Chile}

\begin{abstract}
The present study aims to analyze, from the agency theory, the impact of the dual role phenomenon in the accreditation of professional institutes (PI) and technical training centers (TTC) in Chile. Using a mixed and exploratory research approach, an inspection is performed on the accreditation opinions of $24 \mathrm{PIs}$ and 18 TTCs, which represents $62 \%$ of Chile's professional technical subsystem. The results show evidence of a dual role in seven institutions (17\%), which average 2.1 years of accreditation, a comparatively lower result than that observed in the other accredited institutions assessed. Twelve quantitative institutional accreditation variables are analyzed, verifying that, the observance of a dual role together with four other variables, constitute significant components when explaining the accreditation years of PIs and TTCs in Chile. It is concluded that the PI and TTC dual role phenomenon is a component that leads to tensions and does not guarantee an adequate separation of functions (management and property), which negatively impacts their accreditations.
\end{abstract}




\section{INTRODUCCIÓN}

Las universidades son comprendidas como sistemas sociales abiertos y vivos, que interactúan, influyen y son influidas por el ambiente que las rodea, en una relación dinámica, permanente y recursiva (Dee, 2007). En línea a este precepto, la educación superior chilena en las últimas décadas ha experimentado continuas transformaciones en los ejes que sostienen su estructura, caracterizándose por una masificación y diversidad de la matrícula producto del aumento del número de instituciones de educación superior que lo componen (Espinoza, 2017), transitando desde un pequeño sistema de elites profesionales, intelectuales, académicas, técnicas y políticas, hacia un régimen de masas (Altbach, 2013).

Esta masificación se ha visto expresada en la oferta de carreras en todos los niveles de formación, lo cual introduce diferencias entre instituciones que persiguen objetivos comunes, es decir, alcanzar reconocimiento de la sociedad como entidades que dan garantía y fe pública de la calidad de sus servicios académicos (Scharanger et al., 2010). En cuanto a la calidad, esta se constituye como una dimensión que incorpora diferenciación en sí misma, además de un atributo y valor a los servicios que las organizaciones confieren (Bondarenko, 2007), para lo cual, requieren establecer procedimientos y mecanismos que salvaguarden su actuación en vistas del logro de sus propósitos y fines (Harvey et al., 1993).

En este sentido, la calidad en la educación superior se constata a través de procesos de evaluación externa, específicamente de acreditación, los cuales, se desarrollan mediante la comprobación de un conjunto de criterios predeterminado para obtener un sello de calidad (Lemaitre., 2018). Sobre el particular, en Chile el proceso de acreditación se instaló a partir del año 1999, primeramente a nivel de carreras de pregrado y programas de postgrado, ampliándose el año 2006 a instituciones de educación superior como un proceso voluntario (Ley 20.129), siendo profundizado, como uno obligatorio el año 2018 tras la promulgación de la Ley 21.091 sobre Educación Superior.

En este escenario, donde la acreditación resulta decisiva para las instituciones de educación superior, es pertinente precisar que, en Chile, el sistema de educación superior es de provisión mixta, es decir, coexisten dos subsistemas: el Universitario, y el Técnico Profesional, donde coinciden Institutos Profesionales (IP) y Centros de Formación Técnica (CFT). En tal sentido, se analizaron aquellas variables que podrían explicar la acreditación en los IP y CFT, comenzando con una revisión de las resoluciones de acreditación de 42 IP y CFT, lo que representa un $62 \%$ del sistema, individualizando en 7 de ellas problemas de agencia (Jensen, 1993), vinculado al "doble rol" (Ganga et al., 2016), hallazgo consignado como una debilidad por parte del organismo acreditador, en particular, lo concerniente a la participación del rector como miembro del Máximo Cuerpo Colegiado. Asimismo, se observan diferencias en cuanto a los desempeños obtenidos en años de acreditación, puesto que, los 7 IP y CFT en que se advirtió prominencia del doble rol, promedian 2,1 años de acreditación, mientras que el resto de las instituciones acreditadas, detentan en promedio, 3,9 años.

Sobre la base del hallazgo, se procedió a analizar este factor junto a otras 11 variables empleadas en los procesos de acreditación, precisadas en la metodología del artículo. Por intermedio de un modelo de regresión lineal múltiple, se buscó comprobar la incidencia del doble rol en los años de acreditación de los IP y CFT en Chile. A este respecto, los resultados ponen de relieve que el doble rol, junto a otras cuatro variables, a saber: retención de primer año; académicos Jornada Completa Equivalente (JCE); JCE con grado académico de magíster; y JCE con grado académico de licenciado o título profesional, constituyen variables explicativas de sus años de acreditación.

\section{OTROS ANTECEDENTES}

La Teoría de Agencia principia en el supuesto básico que los propietarios de una organización (Principal), delegan en un tercero (Agente), la responsabilidad de accionar los propósitos y fines del primero para maximizar sus funciones de utilidad. En tal sentido, si ambas partes persiguen maximizar sus beneficios, y bajo el supuesto de que el Agente dispone de más información que el Principal (asimetría de información), existen argumentos para conjeturar, que el Agente, indefectiblemente no actuará procurando el interés supremo del Principal, en consecuencia, estamos en presencia de un problema de agencia (Speer et al., 2014).

La divergencia de intereses y roles entre los actores, así como la coexistencia de objetivos disímiles entre sus actores, son hechos recurrentes en la Teoría de Agencia (Peris et al., 2012). En tal sentido, Edwards (1988), sostienen que sólo hay nociones o creencias sobre cual o cuales son los intereses de ambas partes, lo que se agudiza en el acaecimiento de que el Principal, está compuesto por grupos de individuos heterogéneos, que no dan a conocer con elocuencia los propósitos y fines que persiguen. Por tanto, reconociendo estas disimilitudes desde un enfoque de agencia, las organizaciones suelen constituir órganos de gestión denominados "Consejos de Administración", a fin de controlar y supervisar sus operaciones (Baysinger et al., 1990). 
De acuerdo con lo precitado, si los propósitos y fines no son conocidos a cabalidad por el Agente, comienza una especulación en relación con su rendimiento, así como también, sobre la configuración de sus acciones para el logro de los objetivos organizacionales. A este respecto, autores sostienen que el "Principal" necesariamente deberá emprender acciones tendientes a minimizar el problema de agencia, lo cual supone un "costos de agencia" (Tosi et al., 2003) los que se presentan en tres ámbitos; 1 ) los reembolsos del Principal para monitorear las actividades del Agente, denominados "costos de supervisión"; 2) costos para formalizar el acuerdo contractual, calificados como "costos de transacción"; y 3) las pérdidas de la riqueza del Principal, como efecto de los objetivos divergentes del Agente, apellidado como "pérdidas residuales".

La evolución de los fundamentos teóricos y aplicación de la teoría de agencia ha permitido una diversificación en relación con su área de aplicación, una de estas es la educación superior. En este sentido, destaca la aplicación de la teoría de agencia en trabajos en la relación del profesor-alumno (Smith et al., 2005), y su consideración como variable explicativa de la baja calidad en la educación superior. Por otro lado, el estudio de la relación de agencia ha tenido cabida en las universidades chilenas, donde Ganga et al., (2012), analiza el sistema de compensaciones e incentivos, además de las opiniones de rectores (Agente) y miembros del Máximo Cuerpo Colegiado, tales como Juntas Directivas, Directorios o Consejos Superiores (Principal).

\section{Asimetría de la información}

La asimetría de la información subyace con frecuencia raíz de la disparidad de información que dispone el Principal con relación a la actuación del Agente (Pepper et al., 2015). Sobre este punto, Mackenzie et al., (2013), manifiesta que, cuando ambas partes disponen de información desemejante sobre una transacción, generalmente es el Agente quien domina mayores antecedentes, generando desconfianza e ineficiencia en las transacciones. Asimismo, otros autores han puesto de relieve las alteraciones que ocasiona este fenómeno en las organizaciones, entre ellas, lo conocido como "selección adversa" (Krishna; 2008) y "riesgo moral", esta última, relacionada a las actuaciones futuras del Agente, cuando sus acciones son inobservadas por el Principal, escenario en el cual podría actuar en su beneficio, y no en atención a los objetivos del Principal (Stiglitz et al., 1981).

Así también, se hace ostensible que la asimetría de la información es un hecho habitual, y en consecuencia, se debe procurar aminorar a objeto de asegurar la transparencia en la gestión organizacional (Speer et al., 2014). En este sentido, es plausible que las organizaciones establezcan eficaces mecanismos de control que contribuyan a construir una cultura de la transparencia, que promueva la configuración de información en cantidad y calidad como fundamento para la toma de decisiones (Krishna et al., 2008; Jensen, 1993). Asimismo, es recomendable establecer estrategias y canales de comunicación eficientes que den fluidez a la información entre ambas partes, donde el control del Gobierno Corporativo resulta primordial, en el cometido de encausar los intereses disímiles entre ambos, reduciendo conductas ventajistas (Oster, 1998).

En lo que respecta a la educación superior, las tensiones que se presentan en términos de la asimetría de la información han sido estudiadas por diversos autores, quienes coinciden en que este fenómeno emerge a raíz de que el Máximo Cuerpo Colegiado (Principal), suele solicitar información con escasa frecuencia, donde es probable que el rector (Agente) maneje mayores niveles de información relativa a la gestión de la universidad, y por tanto, el Principal, cuya función puede sintetizarse en el control, la toma de decisiones y la rendición de cuentas, perciben asimetría de información (Ganga et al., 2016). Por su parte, la asimetría de la información también se ha visto expresada en los procesos de acreditación, donde se presenta información de manera "estratégica", relevando algunos antecedentes por sobre el resto (Ewell, 2004).

\section{El doble rol}

El "doble rol", es un fenómeno que se vincula a la integración de roles y funciones del Agente y el Principal. En el caso de las instituciones de educación superior, se advierte en el cometido del rector (Agente), y su participación tanto como Directivo Superior y miembro del Máximo Cuerpo Colegiado, lo cual reduce la independencia y objetividad que debieran tener los directivos en sus decisiones, además de su capacidad de supervisión. Lo anterior, se profundiza en los casos en que el rector, no sólo tiene una participación con derecho a voz y voto, sino que además asume la función de dirigir el consejo (Ganga et al., 2016).

Este fenómeno ha sido sujeto de estudios por diversos autores, quienes recomiendan la necesidad de separar ambas funciones (la propiedad y la gestión), para así no inducir una tensión, que por una parte, reduzca las funciones del máximo directivo, y por otra, merme la independencia del Máximo Cuerpo Colegiado (Berrone, 2009). Para Weisbach et al. (1996), lo planteado, origina una inevitable coacción, dado que, los objetivos de maximización del valor de ambas partes pueden incidir en la viabilidad futura de la organización. Del mismo modo, esta situación tiene repercusiones en la gestión universitaria, y por consiguiente, en la calidad y acreditación, puesto que, se ve disminuida la capacidad de control y supervisión del Máximo Cuerpo Colegiado a las funciones del rector. 


\section{La calidad, su aseguramiento y la acreditación en Chile}

Variadas investigaciones ponen de manifiesto que la calidad resulta un designio complejo de colegir, con diversas acepciones; además de contextual y relativa, es decir, depende de la forma en que es percibida por los diferentes interesados (Mukwambo., 2019). Por otra parte, autores sostienen que la calidad puede ser determinada por la percepción del usuario "calidad percibida" (Douglas et al., 2006), por la excelencia, distinguiéndola como un juicio del usuario respecto a la superioridad del servicio (Rust et al., 1994); y como un elemento de consistencia, en cuanto a la consecución de los propósitos y fines institucionales (harvey et al, 1993).

Claro está que no existe una única adyacencia para definir que es la calidad y su aseguramiento, no obstante, este último concepto, es usualmente empleado para describir un conjunto de mecanismos que propenden al control, la garantía y la promoción de la calidad (Lemaitre et al., 2012; Martínez et al., 2020), los que, resultan pertinentes estructurarlos a través de Sistemas Internos de Aseguramiento de la Calidad que posibiliten su evaluación permanente bajo parámetros comparativos y una retroalimentación sistemática desde y hacia las partes interesadas (Singels et al., 2001). Al mismo tiempo, la calidad en la educación superior se verifica a través de procesos de acreditación, los que persiguen dar garantía ante la sociedad del funcionamiento de una institución, sus objetivos, y las condiciones para prever su consecución (Scharager et al., 2010). A este respecto, para Lemaitre et al., (2018) coexisten dos enfoques para evaluar la calidad; el primero: desde uno interno y retrospectivo, que considera mecanismos de acreditación centrados en la transparencia; y el segundo: centrado en acciones de mejoramiento continuo, de control interno y autorregulación.

En Chile, la complejidad del sistema universitario impulsó la instalación de las bases de la acreditación el año 1999, esto a raíz de la creación de la Comisión Nacional de Acreditación de Pregrado (CNAP) y la Comisión Nacional de Acreditación de Postgrado (CONAP), las cuales, tenían por misión velar por la calidad de las carreras de pregrado y programas de postgrado respectivamente, constituyéndose para estos fines, los primeros criterios y normas para la acreditación en nuestro país. Ambos procesos rápidamente lograron legitimidad y valoración, lo que incitó su ampliación desde las carreras y programas, hacia las instituciones de educación superior. De esta manera, el año 2003 inició un plan experimental y voluntario de acreditación institucional, al cual se sometieron 14 instituciones de educación superior (Cancino et al., 2014). En lo sucesivo, el año 2006 se promulga la Ley № 20.129 sobre Aseguramiento de la Calidad de la Educación Superior, que crea la Comisión Nacional de Acreditación (CNA). Desde entonces, diversos actores sociales y políticos abogaron por la idea de impulsar una amplia reforma a la educación superior, materializándose con un proyecto de Ley ingresado por la Presidencia del país al Congreso de la Nación el año 2016; la cual es promulgada como Ley el año 2018.

\section{METODOLOGÍA}

El enfoque de investigación de este artículo es de carácter mixto y exploratorio. Se analizaron 42 Instituciones de Educación Superior del subsistema técnico profesional, en específico, 24 IP y 18 CFT; lo que representa un $62 \%$ de las instituciones que mantienen registro oficial en el Consejo Nacional de Educación (CNED). Las instituciones que no figuran en la muestra corresponden a aquellas denominadas "fuera del sistema" (CNA, 2020), no presentadas a procesos de acreditación, con excepción del IP INCACEA, IP Galdámez, CFT CEITEC y CFT Barros Arana, de los cuales no se dispuso de información íntegra de sus indicadores. En cuanto al enfoque cualitativo, se realizó una revisión documental de las resoluciones de acreditación de los IP y CFT en estudio, en particular, del último proceso de acreditación que sortearon y que se encuentran contenidas en el sistema de registro documental en la página web de la CNA. Con esta exploración, se precisó conocer el pronunciamiento del organismo acreditador en observancia de un doble rol en dichas instituciones.

En cuanto a las variables para realizar el modelo cuantitativo, estas fueron extraídas desde el Sistema de Información de Educación Superior (SIES) con cohorte del año 2019. Para este objeto, se examinaron 12 variables, las que, en consideración a las 42 instituciones sometidas a análisis, da un total de 559 datos estudiados. A continuación, es posible apreciar las variables atendidas en el estudio: 1) Años de acreditación; 2) Matrícula de pregrado; 3) Retención de primer año; 4) Retención de segundo año; 5) Sobre permanencia en semestres; 6) Académicos Jornada Completa Equivalente (JCE); 7) JCE con grado académico de doctor; 8) JCE con grado académico de magíster; 9) JCE con grado académico de licenciado o título profesional; 10) JCE con título técnico; 11) Estudiantes por JCE; 12) Presencia de doble rol (variable Dummy): 1: Se observa como debilidad en resolución de acreditación respectiva y 0: no se observa como debilidad en resolución de acreditación respectiva. Respecto a las hipótesis a contrastar, estas son las siguientes: i) $H_{0}$ : "La presencia del doble rol no explican los años de acreditación de los IP y CFT en Chile"; y ii) $H_{1}$ : "La presencia del doble rol explican los años de acreditación de los IP y CFT en Chile" 


\section{RESULTADOS}

De las 42 instituciones sometidas a análisis, en 7 de ellas (17\%), fue posible constatar un problema de doble rol. Este hallazgo es relevado como problemático para la calidad de las instituciones, y su expresión se hace patente en los números de años de acreditación obtenidos por éstas, en circunstancias que, los IP y CFT en que se identificó este hecho sus años de acreditación fluctúan entre los 0 y 4 años, siendo 2,1 el promedio. En contraste, los IP y CFT que no poseen vestigio de este suceso, su acreditación promedia los 3,9 años. Las observaciones contenidas en las resoluciones de acreditación de los IP y CFT en los que se comprobó atisbo de doble rol, fue posible precisar lo siguiente:

Tabla 1: Observaciones relacionadas con el doble rol en las resoluciones de acreditación.

\begin{tabular}{|c|c|c|}
\hline Institución & $\begin{array}{l}\text { Años de } \\
\text { Acreditación }\end{array}$ & Observación Resolución de Acreditación \\
\hline IP ARCOS & 4 años & $\begin{array}{l}\text { "Resulta importante para la Comisión mencionar, que los cargos de } \\
\text { autoridades unipersonales debieran tener independencia de la participación } \\
\text { en la Junta Directiva, lo que podría quedar resuelto en el escenario de la } \\
\text { nueva legislación, con la manifiesta intención del Instituto de transformarse } \\
\text { en una institución sin fines de lucro". }\end{array}$ \\
\hline IP ECAS & 4 años & $\begin{array}{l}\text { "En este sentido, si bien la transformación a corporación permite el } \\
\text { desarrollo y funcionamiento de la entidad, es importante que propenda a una } \\
\text { adecuada diferenciación entre la propiedad y la gestión. En opinión de la } \\
\text { Comisión, es indispensable determinar mecanismos que consigan } \\
\text { resguardar la independencia en la toma de decisiones del Instituto". }\end{array}$ \\
\hline IP CIISA & 3 años & $\begin{array}{l}\text { "Se reconocen tres niveles de gestión dentro del Instituto: el nivel } \\
\text { estratégico, el nivel táctico y el nivel operacional. No obstante, estos niveles, } \\
\text { no se observa una diferenciación entre la propiedad del Instituto y su } \\
\text { gestión, y que se establezcan mecanismos que resguarden la } \\
\text { independencia en la toma de decisiones". }\end{array}$ \\
\hline IP CULINARY & 2 años & $\begin{array}{l}\text { "Sin embargo, cabe relevar la importancia de ir transitando hacia una mayor } \\
\text { diferenciación entre propiedad y la gestión, en base a mecanismos que } \\
\text { logren resguardar la independencia en la toma de decisiones, así como la } \\
\text { formalización de mecanismos para declarar y resolver potenciales conflictos } \\
\text { de interés. La preocupación expresada cobra aun mayor relevancia si se } \\
\text { considera el hecho de que el rector es parte de la propiedad de la } \\
\text { Institución" }\end{array}$ \\
\hline CFT IPROSEC & 2 años & $\begin{array}{l}\text { "El Centro es una sociedad de responsabilidad limitada, compuesta por } \\
\text { cinco socios, una de ellas es la rectora actual y otra la anterior rectora, quien } \\
\text { ahora forma parte del Consejo Superior de la Institución" }\end{array}$ \\
\hline CFT IDMA & No acreditado & $\begin{array}{l}\text { "El Centro cambió a su director general decisión requerida por la nueva } \\
\text { conformación de gobierno para establecer límites claros entre la } \\
\text { participación de la propiedad y la conducción de la institución. Dicha } \\
\text { modificación se mantuvo, no obstante, por un período de seis meses tras los } \\
\text { cuales el anterior director asumió sus funciones, con lo cual se volvió a } \\
\text { plantear el conflicto de interés que se presentaba anteriormente" }\end{array}$ \\
\hline CFT CANON & No acreditado & $\begin{array}{l}\text { "La Institución mantiene un sistema de rendición de cuentas para las } \\
\text { autoridades superiores y un informe anual de las sedes como elemento } \\
\text { cuantitativo para la toma de decisiones. No obstante, cabe hacer presente } \\
\text { que las mismas autoridades que deben rendir cuenta son las que componen } \\
\text { los órganos colegiados que reciben la cuenta, lo que, en la práctica, deja } \\
\text { inoperante el mecanismo de control y supervisión que corresponde a las } \\
\text { estructuras colegiadas superiores. De este modo, se concluye que la } \\
\text { Institución carece de mecanismos para declarar y resolver potenciales } \\
\text { conflictos de interés entre dueños y autoridades del Centro" }\end{array}$ \\
\hline
\end{tabular}

Adicionalmente, se procedió a realizar un modelo de regresión lineal múltiple, realizando para ello, las verificaciones de supuestos del modelo mediante la comprobación de la linealidad de las variables a través de diagramas de dispersión. Asimismo, se verificó la normalidad en la distribución de los datos de las variables empleadas a través de los test de "Kolmogorov Smirnov" y "Shapiro Wilk". Por último, se comprobó la homocedasticidad de las varianzas, aplicando para ello el test de "Levene", lo que arrojó, una significancia de $0.3009(p<0,05)$, en consecuencia, se asume homogeneidad. Para la comprobación, se empleó un nivel de confianza del $95 \%$. 
Tabla 2: Análisis de regresión lineal múltiple

\begin{tabular}{|c|c|c|c|c|}
\hline Años de acreditación & Coef. & Std. Error & $t$ value & $\operatorname{Pr}(>|t|)$ \\
\hline (Intercept) & $-0,2875916$ & 1,9110206 & $-0,15$ & 0,8814 \\
\hline Matrícula de pregrado 2019 & 0,0002591 & 0,0002125 & 1,219 & 0,2322 \\
\hline Retención de primer año & 3,2159271 & 1,4136454 & 2,275 & $0,0302^{*}$ \\
\hline Retención de segundo año & 0,5028579 & 0,6999505 & 0,718 & 0,4781 \\
\hline Sobre permanencia (semestres) & 0,0151806 & 0,4329010 & 0,035 & 0,9723 \\
\hline Académicos JCE & 0,0422521 & 0,0494960 & 0,854 & 0,4001 \\
\hline JCE con grado académico de doctor & $-0,0359332$ & 0,4624415 & $-0,078$ & 0,9386 \\
\hline JCE con grado académico de magister & $-0,0545923$ & 0,0455282 & $-1,199$ & 0,2399 \\
\hline JCE con grado académico de licenciado o título profesional & $-0,0569799$ & 0,0419574 & $-1,358$ & 0,1846 \\
\hline JCE con título técnico & $-0,0299750$ & 0,0913892 & $-0,332$ & 0,7452 \\
\hline Estudiantes por JCE & $-0,0012097$ & 0,0101606 & $-0,119$ & 0,9060 \\
\hline Presencia de doble rol & $-1,0076439$ & 0,7581777 & 2,045 & 0,0670 \\
\hline \multicolumn{5}{|l|}{ Signif. Codes: $0^{\star * *{ }^{*}} 0.001^{\star * *^{\prime}} 0.01^{{ }^{* \prime}} 0.05^{`}{ }^{\prime} 0.1^{\prime \prime} 1$} \\
\hline \multicolumn{5}{|l|}{$\begin{array}{l}\text { Residual standard error: } 1.629 \text { on } 30 \text { degrees of freedom } \\
\text { Multiple R-squared: } 0.52 \text {, Adjusted R-squared: } 0.344 \\
\text { F-statistic: } 2.955 \text { on } 11 \text { and } 30 \mathrm{DF}, \mathrm{p} \text {-value: } 0.009064\end{array}$} \\
\hline
\end{tabular}

Para evaluar la bondad de ajuste, en primer lugar, se analizó el coeficiente de correlación de Pearson " $\mathrm{R}^{2 "}$, el cual es de 0.52; por otro lado, el $\mathrm{R}^{2}$ ajustado es de 0.444 , por ende, las variables independientes predicen el $44 \%$ de los años de acreditación de los IP y CFT. A su vez, se analizaron los residuos del modelo aplicando el estadístico Durbin Watson, el cual arrojó un resultado de 1,622; con lo cual, es posible sostener que no existen indicios de autocorrelación serial en los datos estudiados; $1,5<\mathrm{DW}<2,5$. En cuanto al modelo de regresión múltiple, la variable dependiente " $Y$ ", viene dada por: "años de acreditación", en tanto, nuestras variables independientes $(\beta 1 X 1+\ldots .+\beta n X n)$ están representadas por las restantes 11 variables descritas en la metodología del artículo. Sobre el particular, se desprende que los años de acreditación de los IP y CFT se explica por la variable $X 1$ : Retención de primer año, y en consecuencia, se acepta $H_{0}$ : resultando el siguiente modelo (ec. 1).

$$
Y=-0,2875916+0,0302 \times 1
$$

De acuerdo con los resultados obtenidos en el modelo inicial, y con el objetivo de identificar aquellas con mayor eficiencia en la estimación, se procedió a excluir 4 variables, todas ellas con mejor grado de significancia. Al respecto, los resultados obtenidos son los siguientes:

Tabla 3: Análisis de regresión lineal múltiple con variables excluidas

\begin{tabular}{|c|c|c|c|c|}
\hline Años de acreditación & Coef. & Std. Error & $t$ value & $\operatorname{Pr}(>|t|)$ \\
\hline (Intercept) & $-0,3964627$ & 0,906329 & $-0,437$ & 0,66457 \\
\hline Matrícula de pregrado 2019 & 0,0002485 & 0,000129 & 1,926 & 0,06253 . \\
\hline Retención de primer año & 3,3016957 & 0,975675 & 3,384 & $0,00181^{*}$ \\
\hline Retención de segundo año & 0,5275129 & 0,555257 & 0,950 & 0,34880 \\
\hline Académicos JCE & 0,0290559 & 0,015161 & 2,523 & $0,01648^{*}$ \\
\hline JCE con grado académico de magister & $-0,0395641$ & 0,017017 & $-2,325$ & $0,02618^{*}$ \\
\hline JCE con grado académico de licenciado o título profesional & $-0,0454448$ & 0,016291 & $-2,790$ & $0,00859^{* \star}$ \\
\hline Presencia de doble rol & $-1,0133910$ & 0,667442 & $-2,118$ & $0,04781^{*}$ \\
\hline \multicolumn{5}{|l|}{$\begin{array}{l}\text { Residual standard error: } 1.535 \text { on } 34 \text { degrees of freedom } \\
\text { Multiple R-squared: } 0.5171 \text {, Adjusted R-squared: } 0.4176 \\
\text { F-statistic: } 5.2 \text { on } 7 \text { and } 34 \text { DF, p-value: } 0.0004237\end{array}$} \\
\hline Signif. Codes: $0^{\star * * * \prime} 0.001^{\star * *^{\prime}} 0.01^{\star * \prime} 0.05^{\prime} .^{\prime} 0.1^{\prime \prime} 1$ & & & & \\
\hline
\end{tabular}

En este modelo, el $R^{2}=0.5171$; y el $R^{2}=0.4176$, considerado como moderado. Asimismo, fue posible concertar, que la bondad de ajuste del modelo no se vio afectada con la exclusión de variables menos significativas. Análogamente, el modelo advierte que la variable dependiente $Y=$ años de acreditación, es explicada por cinco variables independientes, entre ellas, el doble rol, a saber: $X 1=$ Retención de primer año; $X 2=$ académicos JCE; $X 3=\mathrm{JCE}$ con grado académico de magíster; $X 4=\mathrm{JCE}$ con grado académico de licenciado o título profesional; $y, X 5=$ Presencia de doble rol. Por consiguiente, se rechaza $H_{0}$, configurando el modelo de acuerdo a la ecuación 2.

$$
Y=-0,3964627+0,00181 X 1+0,01648 \times 2-0,02618 X 3-0,00859 \times 4-0,04781 \times 5
$$




\section{DISCUSIÓN}

Los resultados obtenidos permiten sostener que la acreditación de los IP y CFT en Chile son explicadas por cinco variables, entre ellas, el fenómeno del doble rol. En tal sentido, esto sugiere una relación inversa a la acreditación institucional, es decir, una menor prominencia del doble rol se traduce en una mayor valoración en términos de calidad. Este hallazgo es congruente con estudios realizados en el sector de la educación superior (Mackenzie et al., 2013; Ganga et al., 2016), además de otros sectores industriales, quienes sugieren que en las organizaciones debe existir una adecuada separación entre la propiedad y la gestión (Berrone, 2009).

Otro elemento a destacar como factor significativo al momento de explicar la acreditación, es la retención; por tanto, una mayor sujeción de estudiantes es un componente que predomina al momento de evaluar la calidad de un IP y CFT. En vista del panorama nacional, destaca que los IP y CFT tienen en promedio una deserción del $35 \%$ anual; $67 \%$ y $64 \%$ respectivamente, mientras que las universidades presentan en promedio un $21 \%$ de deserción (CNED, 2019). Este hallazgo es cohesivo con diversas investigaciones que instalan la deserción como un elemento sensible, que supone rasgos de ineficiencia del sistema educacional y que tiene impacto en la gestión de las instituciones (Himmel, 2002). Al respecto, esta variable tiene diversas aproximaciones que no solo se vinculan a asuntos de rendimientos académicos de los estudiantes, sino que a un conjunto de enfoques del tipo psicológico (Metz, 2004), sociológico (Braxton et al., 1997), y económico (Ishitani et al., 2002).

Así también, se observa como significante tres variables que pueden ser combinadas, a saber: Académicos JCE, JCE con grado académico de magíster, además de aquellos JCE con grado de licenciado o título profesional. En el caso de las JCE, se observa que una mayor dedicación de académicos supone un componente positivo al momento de explicar los años de acreditación de los IP y CFT, en contraste, se advierte que una menor dedicación de académicos con título profesional, grado de licenciatura o magíster, es congruente con una mayor valoración de calidad representada en años de acreditación. Este hallazgo además tiene sustento empírico, toda vez que, en el país, los CFT presentan una relación promedio de 47 estudiantes por JCE, mientras que el 4,1\% de la composición de JCE cuenta con estudios de postgrado. En el caso de los IP, estos presentan una relación de 41,1 estudiantes por cada JCE, y un 15,1\% de sus académicos JCE poseen postgrado. Lo anterior, resulta disímil con el subsistema universitario, el cual en promedio cuenta con 25 estudiantes por JCE, además de un 66\% de académicos postgraduados (CNED, 2019).

\section{CONCLUSIONES}

De acuerdo con el trabajo presentado y los resultados obtenidos, se concluye lo siguiente:

1) El fenómeno del doble rol en los IP y CFT, traducido en la participación del rector (Agente) en el Máximo Cuerpo Colegiado (Principal), es un componente que conduce a tensiones y no garantiza una adecuada separación de funciones (gestión y propiedad), incidiendo negativamente en su acreditación.

2) La retención de primer año constituye un elemento indicativo de la calidad de los IP y CFT, por tanto, una mayor permanencia de estudiantes en sus programas de estudios influye positivamente en la acreditación.

3) Se percibe que la permanencia de académicos expresadas en JCE, así como la dedicación y cualificación de estos en términos de su título profesional y grado académico de licenciado y magíster, configuran variables explicativas de la acreditación en los IP y CFT. En tal sentido, resalta una relación positiva entre la cantidad de JCE y la acreditación, esto quiere decir, que una mayor dedicación de académicos en estas instituciones es positiva términos de calidad. No obstante, se advierte una relación lineal inversa entre la variable de dedicación en JCE con las cualificaciones señaladas y la acreditación, por tanto, una menor especialización del cuerpo académico no constituye un hecho sustantivo en el cometido de lograr una mayor certificación.

\section{REFERENCIAS}

Baysinger, B., y Hoskisson, R. the Composition of Boards and strategic Control: effects on Corporate strategy. Academy of Management Review, https://doi.org/10.2307/258106 (15), 72-87. (1990).

Berrone, M. Estudio sobre la Estructura de los Consejos de Administración. Una visión contrastada entre Inversores Institucionales y presidentes. IESE Business School y Russell Reynolds Associates. (2009).

Braxton, J., Sullivan, A.,y Johnson, R. Appraising Tinto's theory of college student departure. En. J. Smart (Ed.), Higher Education Handbook of theory and research, doi: 10.12691/education-2-6, (13) 107-164 (1997).

Cancino, V., y Schamal S. Sistema de Acreditación Universitaria en Chile: ¿Cuánto hemos avanzado? Estudios pedagógicos, http://dx.doi.org/10.4067/S0718-07052014000100003., 40(1), 41-60 (2014). 
Dee, T., y Brian, A. Do high school exit exams influence educational attainment or labor market performance. National Bureau of Economic Research, DOI: 10.3386/w12199, NBER working paper N. 12199. (2007).

Douglas, J., Douglas, A., y Barnes, B. Measuring Student Satisfaction at a UK University. Quality Assurance in Education, doi: 10.1108/09684880610678568, (14), 251-267 (2006).

Edwards, J. evolución reciente de la teoría Financiera de la empresa (ii Parte). Revista de Economía, (2145),111-126. (1988).

Espinoza, O. Acceso al sistema de educación superior en Chile. El tránsito desde un régimen de elite a uno altamente masificado y desregulado. Universidades, (74),7-30. ISSN: 0041-8935. (2017).

Ewell, P. Accreditation and the provision of additional information to the public about institution. Council for Higher Education Accreditation,1-11. (2004).

Ganga, C., y Burotto, J. Sistemas de compensación e incentivos: opinión de rectores y miembros de los máximos cuerpos colegiados de las universidades chilenas, Rev. Gaceta Laboral, ISSN: 1315-8597, 18(1),57-85 (2012).

Ganga, C., y Maluk, S. Opiniones de expertos sobre el problema de doble rol en las universidades ecuatorianas. Educação, Ciência e Cultura, doi: 10.18316/2236-6377.16.32., (21) 57-73 (2016).

Himmel, E. Modelo de análisis de la deserción estudiantil en la educación superior. Calidad en la Educación, http://dx.doi.org/10.31619/caledu.n17.409., (17), 91-108 (2002).

Ishitani, T., y Stephen, D. A Longitudinal Investigation of Dropout from College in the United States, Journal of College Student Retention, https://doi.org/10.2190/v4en-nw42-742q-2ntl., 4(2), 173-201 (2002).

Jensen, M. The modern industrial revolution, exit, and the Failure of Internal Control Systems. The Journal of Finance., (48), 831-880. (1993).

Krishna, V., y Morgan, J. Contracting for Information under Imperfect Commitment. The Rand Journal of Economics, https://doi.org/10.1111/j.1540-6261.1993.tb04022.x., 39 (4), 905-925 (2008).

Lemaitre, M. Mecanismos de aseguramiento de la calidad: respuestas a los desafíos del cambio en la educación superior. Calidad en la Educación. https://doi.org/10.31619/caledu.n21.323., (21), 87-106 (2004).

Lemaitre, M.J., y Zenteno, M.E. (editoras). Aseguramiento de la calidad en Iberoamérica. Educación Superior. Informe 2012, 1aㅡ edición, 23-71. CINDA-UNIVERSIA, Santiago de Chile (2012).

Mackenzie, T., Buitrago, Q, y otros tres autores. La teoría de la agencia. El caso de una universidad privada en la ciudad de Manizales, https://doi.org/10.19052/ed.2308, Equidad Desarrollo., (19) 53-76 (2013).

Martínez, E., y Sjoberg, O. Determinación de variables y dimensiones claves para el aseguramiento de la calidad y su impacto en la acreditación de las Universidades chilenas. Rev. Espacios, ISSN: 0798-1015, 41(11), 20-33 (2020).

Metz, G. Challenge and changes to Tinto's persistence theory: A historical review, https://doi.org/10.2190/m2cc-r7y1wy2q-upk5., Journal of College Student Retention, 6(2), 191-207 (2004).

Mukwambo, P. Quality in Higher Education as a Tool for Human Development: Enhancing Teaching and Learning in Zimbabwe, $1^{\text {a }}$ edición. https://doi.org/10.4324/9780429490880., Routledge (2019).

Oster, S. Executive compensation in the nonprofit sector. Nonprofit Management and Leadership, https://doi.org/10.1002/nml.8301., 8(3), 207-221 (1998).

Pepper, A., y Gore, J. Behavioral agency theory new foundations for theorizing about executive compensation. Journal of Management, https://doi.org/10.1177/0149206312461054., 41(4), 1045-1068 (2015).

Peris-ortiz, M., Rueda, C., Souza, G., y otros dos autores. Fundamentos de la teoría organizativa de agencia. Rev. de Economía ICE. Nuevas Corrientes de Pensamiento Económico, ISSN: 0019-977X, (865),107-118. (2012).

Rust, R., y Oliver, R. Service Quality: New Directions in Theory and Practice, http://dx.doi.org/10.4135/9781452229102.n1., sage publications, 1-20 (1994).

Scharager, J., y Aravena, M. Impacto de las políticas de aseguramiento de la calidad en programas de educación superior: un estudio exploratorio. Calidad en la Educación, https://doi.org/10.31619/caledu.n32.150., (32), 16-42 (2010).

Stiglitz, J., y Weiss, A. Credit Rationing in Markets With Imperfect Information. American Economic Review. 71. 393-410. (1981).

Singels, J., Ruél, G., y Van de Water, H. "ISO 9000 series certification and performance", International Journal of Quality \& Reliability Management, doi: 10.1016/j.jpe.2015.01.012., 18 (1), $62-75$ (2001).

Smith, M., Zsidisin, G., y Adams, L. An agency theory perspective on student performance evaluation. Decision Sciences Journal of Innovative Education, https://doi.org/10.1111/j.1540-4609.2005.00051.x., 3(1), 29-46. (2005).

Speer, J., y Hanisch, M. ¿Puede la gobernanza participativa superar asimetrías de información en los mercados políticos rurales? Rev. Política y Gobierno, ISSN: 1665-2037, 21(1), 127-158 (2014).

Tosi, H.,y Brownlee, A. empirical exploration of decision making under agency controls and stewardship structure. Journal of Management Studies, doi: 10.1046/j.1467-6486.2003.00411.x., (40), 2053-2071. (2003). 